\title{
The American Journal of Obstetrics and Gynecology
}

\begin{tabular}{lll}
\hline Vol. II. & St. Lovis, OCTOBER, 1921 & No. 4 \\
\hline
\end{tabular}

\section{Original Communications}

\author{
PNEUMOPERITONEUM AND ROENTGENOLOGY AS AIDS \\ TO MORE ACCURATE OBSTETRIC AND \\ GYNECOLOGIC DIAGNOSIS* \\ By Reuben Peterison, M.D., Ayn Arbor, Mich. \\ From the Department of Obstetrics and Gynecology, University of Michigan.
}

T IS the purpose of this communication to give the results of some 1 ten months' use of roentgenography after the injection of the pelvic and abdominal cavities with gas. Beginning the work with doubt and misgivings as to freedom from danger and practicality from the diagnostic standpoint, it can be emphatically stated that at the present time doubts have been cleared away and that it is one of the most useful procedures ever introduecd into the elinic. Lilio all roentgono graphic procedures, it requires long and close study before the plates of the pelvic and abdominal organs can be correctly interpreted. It was an easier matter after some experimenting to secure good plates from a technical standpoint, than it was to interpret what the plates showed.

Hence it follows that the obstetrician and gynecologist in order to obtain the quickest and most accurate returns from his work with pneumoperitoneum, must associate himself with an expert roentgenologist. Moreover, the latter must be interested in this new field and be ready to devote the necessary time to the interpretation of plates the like of which he may never have seen before. In fact.it must be leam work from the outset. To the gynecologist with his surgical expe-

* Read at the Forty-sixth Annual meeting of the American Gynecoiogical Society, Swampscott, Mass., June 2-4, 1921.

Note: The Editor accepts no responsibility for the riews and statements of authors as published in their " Original Communications."

The papers ineluded in the Transactions of the American Gynecological Society are printed in the order of their presentation. 
rience can be safely entrusted the extremely simple technic of gas inflation, either through the uterus or the abdominal wall. He can be instructed even in the methods of making the plates. It is asking too much, however, to expect the clinician at the outset to interpret what is depicted on the plates. His work and experience have not been along these lines, for he has had to employ the x-ray but seldom in his work.

Personally I am very fortunate in having associated with me in this pneumoperitoneal x-ray work my colleague, Doctor James G. Van Zwaluwenburg, without whose cooperation nothing conld have been accomplished. Not only has he willingly spent many hours studying out the meaning of the various pelvic shadows, bringing into play his

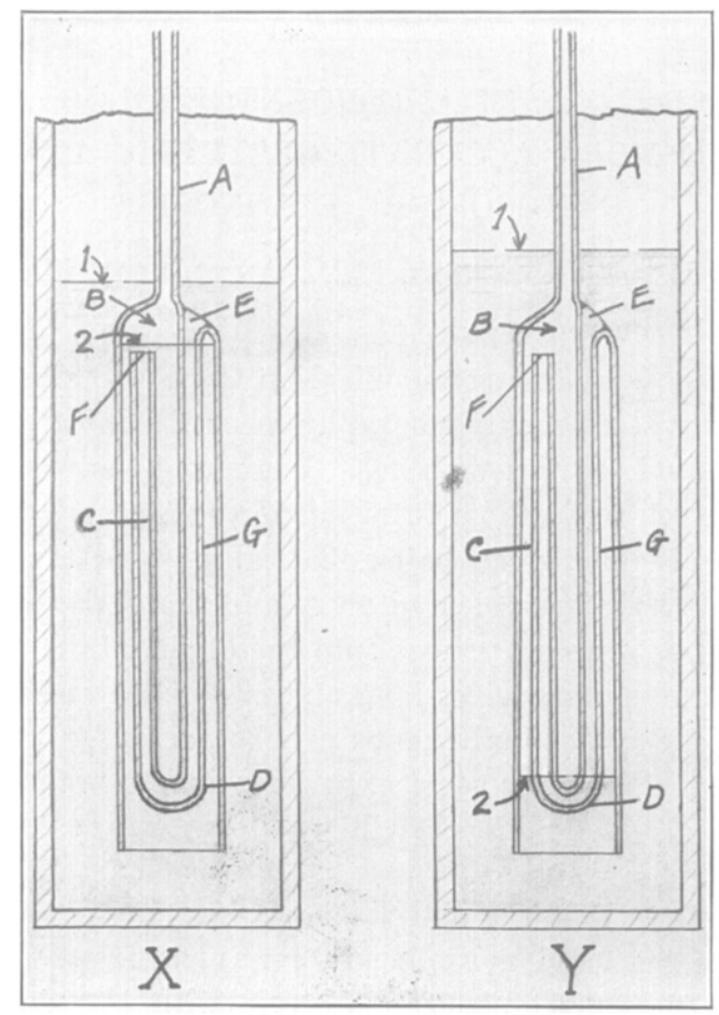

Fig. 1.-Syphon meter.

accurate and comprehensive knowledge of roentgenography, but his patience has been unfailing in guiding me along hitherto unknown paths.

When wo began this work Doctor Van Zwaluwenburg and I agreed that elaborate, complicated, clinical technic must be avoided, if a method was to be developed available for the average diagnostician and clinic. Hence the gas inflation, practically from the beginning, has been made in the gynecologic examining room of the University Hospital. 
Aside from the emptying of the lower bowel and bladder, the patient needs no special preparation for the gas inflation. In a few of our early cases when oxygen gas was employed, the patient was fortified by a hypodermic of morphine, but this is neither necessary nor expedient when the quickly absorbed carbon dioxide gas is used. Moreover, the use of morphine is inadvisable in the case of ambulatory patients who form quite a proportion of this material at the clinic, since out-as well as in-patients are inflated for diagnosis.

No expensive or elaborate table is necessary for gas inflation, merely one where a moderate tilting of the body downward can be secured. We have found this inclined position to be absolutely essential for the

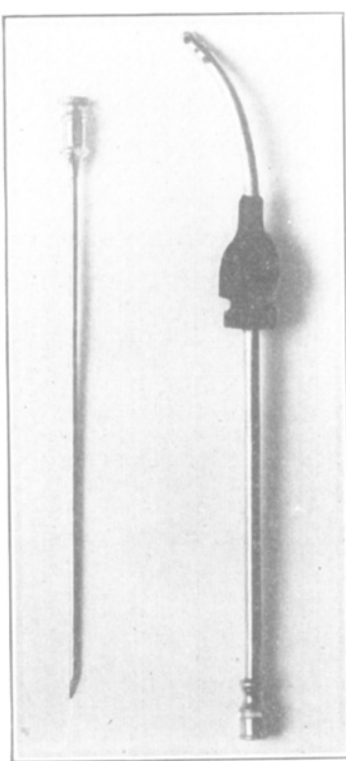

Fig. 2.

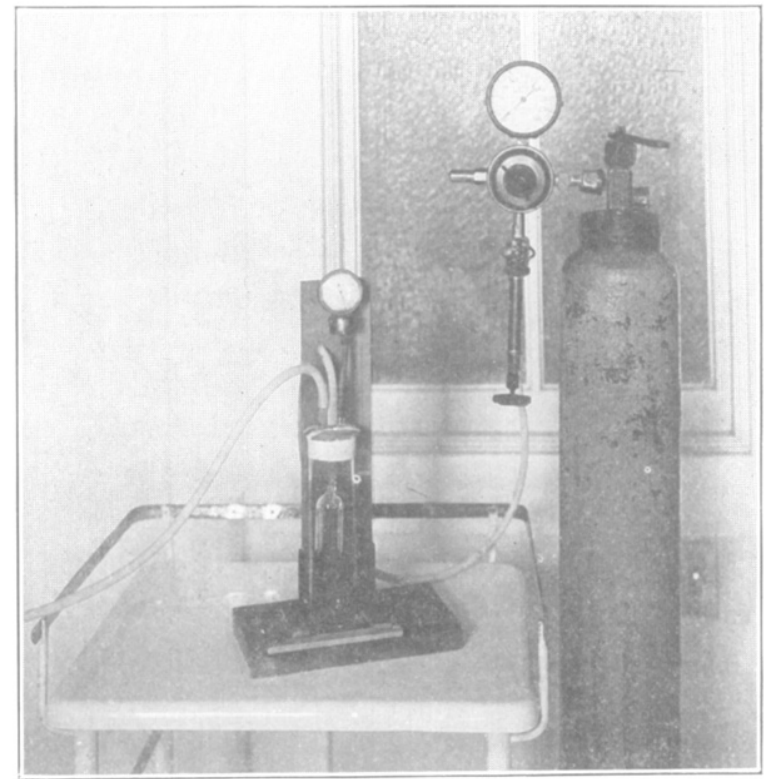

Fig. 3.

Fig. 2.-Needle and uterine cannula.

Hig. 3.-Carbon dioxide tank, gas pressure valve, syphon meter and manometer.

best results, since it is necessary for the pelvis to be freed from bowel coils if the pelvic structures are to be shown. This can be easily brought about by the use of the modified Trendelenburg posture with the patient in the prone position. One such table will suffice, although in the clinic it has been found more convenient to make the injection, either through the uterus or abdomen, on the ordinary examining table, transferring the patient to the ulher table for the x-ray of the pelvis.

At first we used the method of Stein and Stewart, employing a bag between the tank and the point of injection, forcing the gas through the needle by pressure on the bag. This method was aban- 
doned in favor of Rubin's apparatus by means of which the gas can be passed under a pressure which is easily gauged, while the amount injected can be accurately measured.

The apparatus for measuring the gas is an inverted syphon pulsating meter used by the engineers for the measurement of chlorine gas. It is composed of an inverted glass syphon inside a cylindrical glass meter (Fig. 1). The operation of the meter is as follows: "When the downward flowing gas in $A$, reaches the point $D$, it will rush up through the tube $G$ of the syphon and the bell or compartment $B$ will refill with water up to the upper end of $C$. This completes one pulsation or measure of the meter and the amount of gas delivered by this one pulsation is, of course, the capacity of the compartment $B$ between the points $F$ and $D . "$ (Wallace and Tiernan Co.) With this apparatus connected with the gas tank it is only necessary to keep track of the number of pulsations to determine the amount of gas which is flowing through the cannula or needle provided there be no leakage. Any resistance to the gas flow can be readily determined by connecting the cylindrical glass-meter with a manometer. (Fig. 3.) For example, the manometer does not register when the gas is passing through the rubber tube connected with the needle with which the inflation is to be made through the abdominal wall. If, however, the tube be compressed at any point, the manometer index will rapidly rise according to the degree of compression of the tube and fall when the pressure on the tube is released. The same thing occurs when the flow of gas is impeded after the passage of the cannula into the uterus or the needle through the abdominal wall.

It is evident that before passing the gas into the abdomen or pelvis, it is necessary to regulate the amount of gas issuing from the tank under great pressure. We have had great difficulty in regulating the flow with the ordinary levers or wheels with which the tanks are provided. It really required more knowledge and experience to move the lever of the tank to secure the requisite gas flow than to use the cannula or needle. This difficulty has been done away with and the whole inflation made simple, easy and accurate by the use of a gas pressure reducing valve by means of which the flow of gas from the tank can be easily regulated. (Fig. 3.)

The uterine cannula is of metal of the Keyes-Ultzmann type perforated at the tip and provided with a rubber obturator for closure of the external os after the passage of the cannula so as to prevent the escape of the gas backward into the vagina. (Fig. 2.) The ordinary spinal puncture needle is well adapted for the perforation of the abdominal wall and the inflation of the abdominal cavity. (Fig. 2.)

The instruments necessary for the gas inflation are scrupulously sterilized before use. The abdominal wall or in case the transuterine 
route be employed, the cervix, especially in the region of the external os, is prepared by the free use of iodine. Rubber gloves, sterile towels, etc., are employed just as in any surgical procedure. Unless these precautions be taken, it is easily conceivable that infection may follow gas infiation and not be due to any irritation set up by the gas itself. In order to settle the question whether oxygen or carbon dioxide gas as furnished in the ordinary tanks, contained bacteria, as has been claimed, numerous bacteriologic tests were made of these gases as they cmerged from needle or cannula. In every instance the eultures showed no growths. It has been asserted that the low temperature of the gas as it issues under pressure from the tank gives rise to peritoneal irritation. This does not hold true for gas passing through the rubber tubes, because the temperature of this gas as it issues from the needle or cannula was always found to be that of the examining room.

Obviously one must use common sense in the selection of patients to be subjected to gas inflation. Up to the present time no patient with acute inflammatory pelvic disease has been inflated by either route. When the history of recent inflammatory discase is doubtful, even should the patient have a normal temperature, no chances are taken, but the inflation is conducted by the transperitoneal route. While neither shock nor depression have followed the gas inflation, it has been refrained from in certain individuals with impairment of the eirculation. In other words the watchword of the clinic in this work has been to take no chances but to always be on the side of safety.

In none of the 300 cases of gas inflation has there been any sign of peritoneal irritation. Thus we think we are justifier in the conclusion that with the proper selection of cases and with the proper technic there is no danger attached to gas inflation.

The transuterine is the method of choice since it throws much light upon the patency or nonpatency of the fallopian tubes. The gas can be introduced with as little discomfort to the patient by this as by the transperitoneal route. Where the tubes are found impermeable, the gas is introduced through the abdominal puncture. If the gas be passing freely through the tubes into the pelvic cavity, it is our custom to allow enough to flow so that a satisfactory x-ray of the pelvis can be obtained. In other words, the procedure of Rubin for determining the permeability of the tubes with small quantities of gas, has been carried a step further in case the tubes be patent. This has been done deliberately because of the impossibility with present methods of determining whether the gas be passing through one or both tubal openings. In case one tube be diseased and the other open, the condition of the pathologic tube will be shown by the pelvic $\mathrm{x}$-ray. 
The Sims has been found preferable to the lithotomy position where the gas is passed by the transuterine route. (Fig. 4.) The cervix is exposed after the introduction of the speculum and the anterior lip of the cervix seized by the tenaculum forceps after the cervix and adjacent portions of the vagina have been sterilized by iodine. Care is taken to remove all secretions from the cervical canal, a short distance from the external os. The general direction of the uterine canal is located by the sound and the uterine cannula passed, the rubber obturator being pressed within the canal until a snug fit is secured.

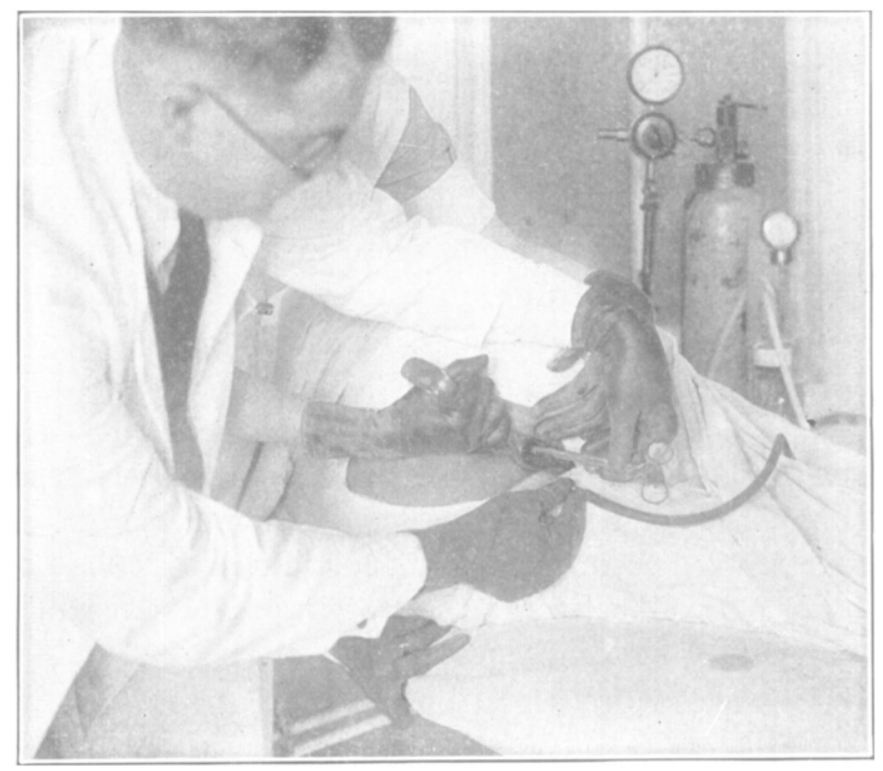

Fig. 4.-Method for transuterine gas inflation. Patient in Sims $^{\wedge}$ position, perineum retracted, tenaculum torceps on anterior cervical lip, cannula in uterus.

Dependent upon the condition of the eervix, more or less outward pressure must be exerted upon the tenaculum, else the gas will eseape backward into the vagina. It must not be forgotten to test the permeability of the camnula before its introduction. In case of blockage, the manometer index will quickly rise, showing that the instrument is faulty. Failure to observe this simple precantion for both cannula. and needle may lead to wrong deductions.

The apparatus should have been previously set by means of the gas pressure valve for about six or eight pulsations a minute. Since the average gasometer of this type measures about 25 cubic centimeters of gas to each pulsation, the apparatus set at this rate of flow will allow of the passage of 125 to 200 cubic centimeters per minute. This amount should be used as a standard, as it has been determined by experience that this is about the maximum quantity which should be passed through normal tubes in one minute. 
If the tube or tubes be permeable after the gas has been allowed to flow through the cannula there will be a rather rapid rise of the manometer to about 100 or 110 . Then as the gas continues to flow there will be quite a sharp drop from 30 to 50 points, at which place the manometer index remains quite steady. On the other hand, if there be any resistance to the flow of gas through the tubes, there will be a steady rise of the manometer and the flow of gas should be stopped at the 200 point. At first we believed that if the gas did not pass at 150 after two or three trials it could be coneinded that the tubes were closed. However, further experience, especially with patients under anesthesia, has shown that patent tubes may require the 200 pressure repeated four or five times before the gas will pass. Whether this means that some obstruction exists in these cases at the isthmic portion of the tube has not been determined.

At first we used too large quantities of gas, some two or three liters, with corresponding pain and discomfort to the patient. Experience has shown that this quantity of gas is unnecessary, if the patient's pelvis be raised and the table tilted so that the gas can rise in the pelvis. (Fig. 5.) This is accomplished after the proper amount of gas has been introduced by holding the patient on the tilting table face downward in the knee-chest position until an inclined board can be placed beneath the thighs. This board makes an angle of about 28 degrees with the plane of the table. Its upper portion is cut out and rounded so that the pubes come to lie just above the deepest portion of the notch. A plate changing tunnel is then placed horizontally on the table and the table tilted forward, the patient prevented from slipping by means of shoulder straps.

Experience has shown that to get the best results the table should be inclined at an angle of about 20 degrees. Excessive inclination of the table, when the pelvic organs are floated forward by the gas, causes too great displacement and makes the pelvic x-ray confusing.

A Coolidge portable unit operating on the ordinary lamp circuit furnishes the x-ray, the direction of the ray being in the long axis of the pelvis and perpendicular to the plate. (Fig. 6.) An 18-inch square of opaque fabric with a $6 \frac{1}{2}$ inch circular hole cut out of its center is laid on the patient's buttocks and serves as a diaphragm. Double screened films are used and the exposure varies from 14 to 20 seconds. The tube shift is in the long axis of the body and the stereo set so produced is used. as though the patient were lying on the right side.

The best results so far as the x-ray plates are concerned, taking into consideration the comfort of the patient, are obtained by the use of from 800 to 1000 cubic centimeters of gas. Larger quantities of gas quickly injected cause great discomfort and even pain to the patient. 
Hence it has been our constant aim to reduce the amount of gas injected to a minimum, our efforts being greatly aided by the use of the table arrangement just described. Since the patients complain of a sense of fullness in the lower abdomen and some discomfort when only 300 to 400 cubic centimeters of gas have been introduced and since that quantity has been found too small for proper inflation, it is obvious that a certain amount of discomfort will always be present. However, the sensation, excluding highly nervous women, may be described as discomfort and not the pain which always accompanies overinflation.

At first we used oxygen gas but abandoned it because it was slowly absorbed with corresponding persistence of the discomfort. Deflation

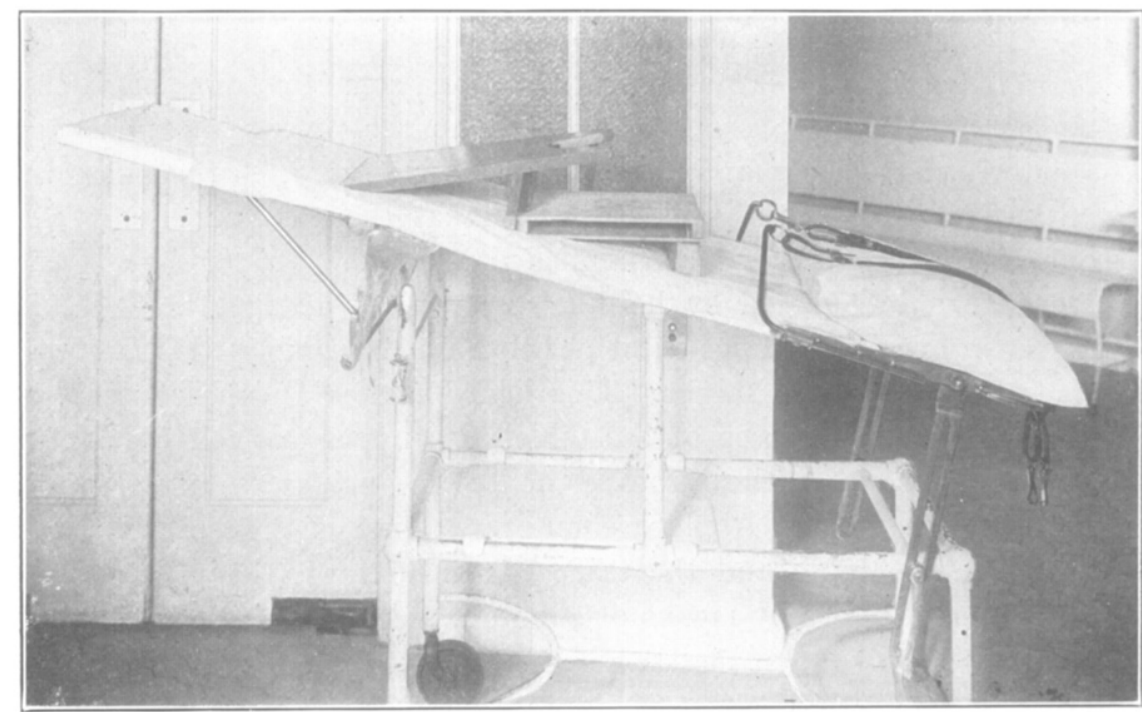

Mig. 5.-Table for taking pelykogrank, showing notched inclined plane, tunneled plate hoider and table at proper inclination $\left(20^{\circ}\right)$

relieved to some degree the symptons but neeessitated a second needle puncture, which for obvious reasons did not improve the technic. For these reasons we substituted for the oxygen, carbon dioxide gas which is absorbed so far as symptoms of discomfort are concerned in from twenty mimutes to one-half hour. After inflation with this gas patients return to the ward or leave the hospital without discomfort a half hour after the plates have been taken. In fact the one disadvantage of this gas is the necessity of having the technic of plate taking work perfectly smoothly. Delay beyond a certain point means so much carbon dioxide gas absorption as to materially lessen the chances of good roentgenograms.

In addition to acute pelvic disease, profuse purulent and bloody uterine discharges are considered contraindications to the passage of 
the gas by the transuterine route. In such cases the gas is passed through the abdominal wall by the following technic: The skin of the abdominal wall from the umbilicus nearly to the pubes and for a width of eight inches is sterilized with iodine. A fold of the abdominal wall just below the navel is compressed laterally with the left hand and raised upward and made tense. A spinal puncture needle is passed through this fold in a slightly upward direction at a spot in the median line one and one-half inches below the umbilicus. (Fig. 7.) If the skin be made tense and the needle be sharp, no preliminary anesthesia of the skin will be necessary. The needle is passed downward

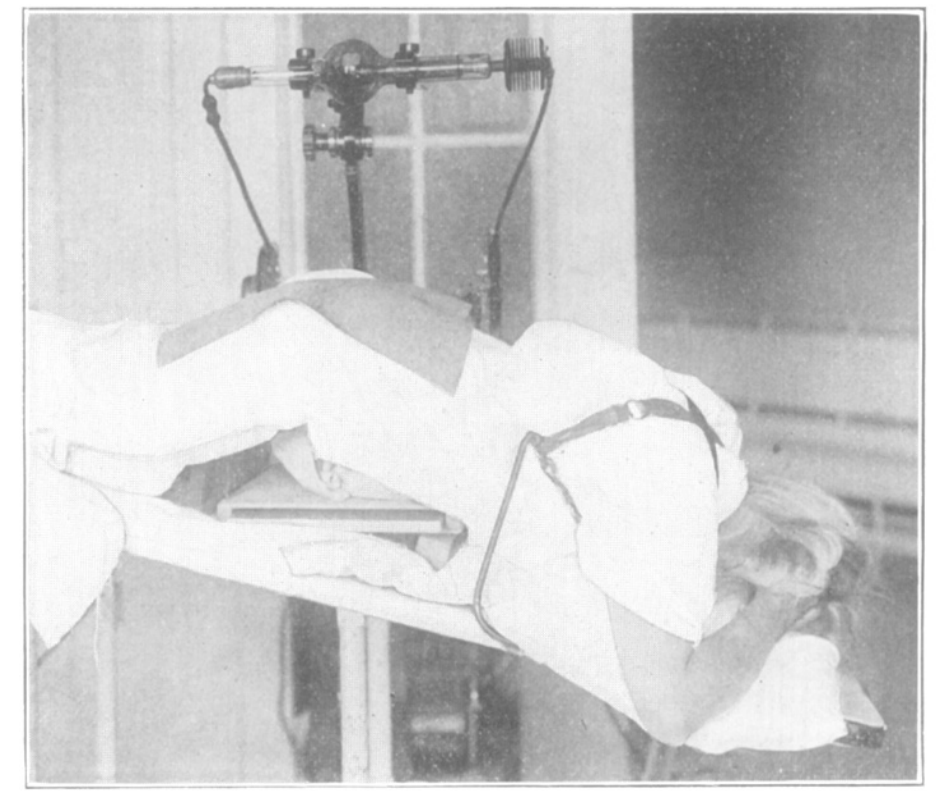

Fig. 6.-Paticnt after inflation in pronc (partial knce-chest) position on inclincd table. Opaque diaphragm, Coolidge portable unit arranged so that ray will fall perpendicular to pelvis and jlate.

until it meets the resistance of the fascia, the left hand still keeping tense the fold of the abdominal wall. The needle is then thrust through the fascia and peritoneum, a little experience giving accurate information to the hand when the peritoneum has been pierced. The stilet is then withdrawn from the needle and the latter connected with the rubber tube leading from the syphon meter. The latter is set by means of the pressure valve to about ten pulsations to the minute, so that it will require approximately four minutes at the rate of 25 cubic centimeters of gas at each pulsation for 1000 cubic centimeters of gas to pass into the peritoneal eavity.

The objection that in case the needle does not pierce the peritoneum, the gas may be forced through the subcutaneous tissues is not valid, 
since by the manometer it can be told at once whether the gas is flowing freely into the cavity or is meeting with an obstruction. When the needle has penetrated the peritoneum the manometer index rises first some 15 or 20 points but falls usually immediately to almost zero. In case this fall does not occur the patient should be directed to take a deep breath which will have the effect of lifting the point of the needle from anything which may be obstructing it.

At first the skin at the point of puncture was anesthetized by Schleich's solution but this bas been discontinued since it was found that very nearly as much pain was produced by this procedure as by the passage of the needle. Obviously if the point of election for the puncture is over what may be an adherent intestine or if a neoplasm

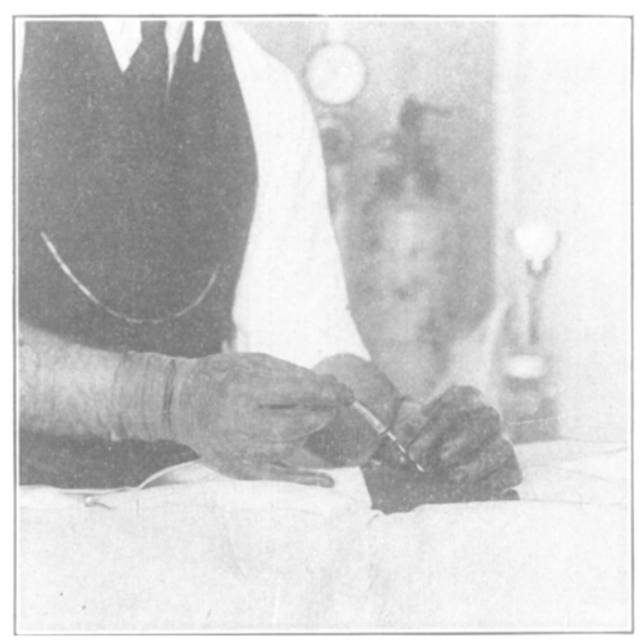

Hig. 7.-Transperitoneal gas inflation. Needle in abdominal cavity and gas flowing.

lies beneath this site, another portion of the abdominal wall must bo chosen.

Before the patient is inflated in addition to a careful history of her case she is subjected to a most complete bimanual pelvic examination to which is added a rectovaginal examination if the case seems to call for it. Without going into details it will suffice to say that an attempt is made in every case to record on the examination sheet what the pelvis contains, not in general but in specific terms. For example, if in the case of inflammatory disease to the left of the uterus the ovary and the tube can be made out, it is so stated. If neither can be recog. nized by the examining finger it is so recorded. The same holds true regarding adhesions, relation of neoplasms to the uterus, ets. In other words such terms as sensitive mass, boggy mass, left-sided inflammatory have been discarded since our work has been checked up 
by the x-ray. We may and do make mistakes but at least they are definite errors from which valuable lessons can be learned.

The plates are taken to the $x$-ray laboratory and interpreted by Doctor Van Zwaluwenburg withont, reference to the clinical findings. Then the clinieian and roentgenologist go over the plates set up in stereo in an endeavor to reconcile the two findings. If the case be operative, the patient is carefully examined under anesthesia and the

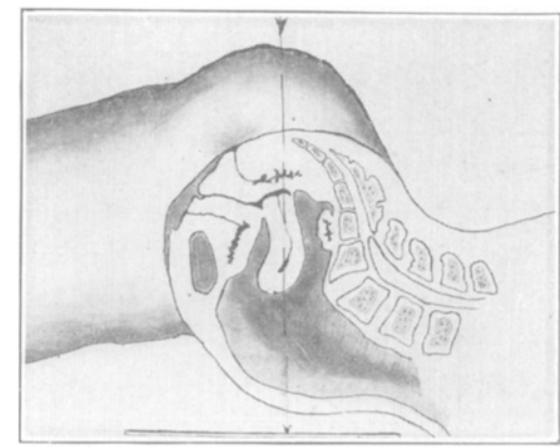

Fig. 8.- Correct inclination of patient, $x$-ray in axis of pelvis and perpendicular to plate In this position with movable uterus the anterior and posterior culdesacs will be filled with gas. Plate will show cross sections of istbmus and fundus.

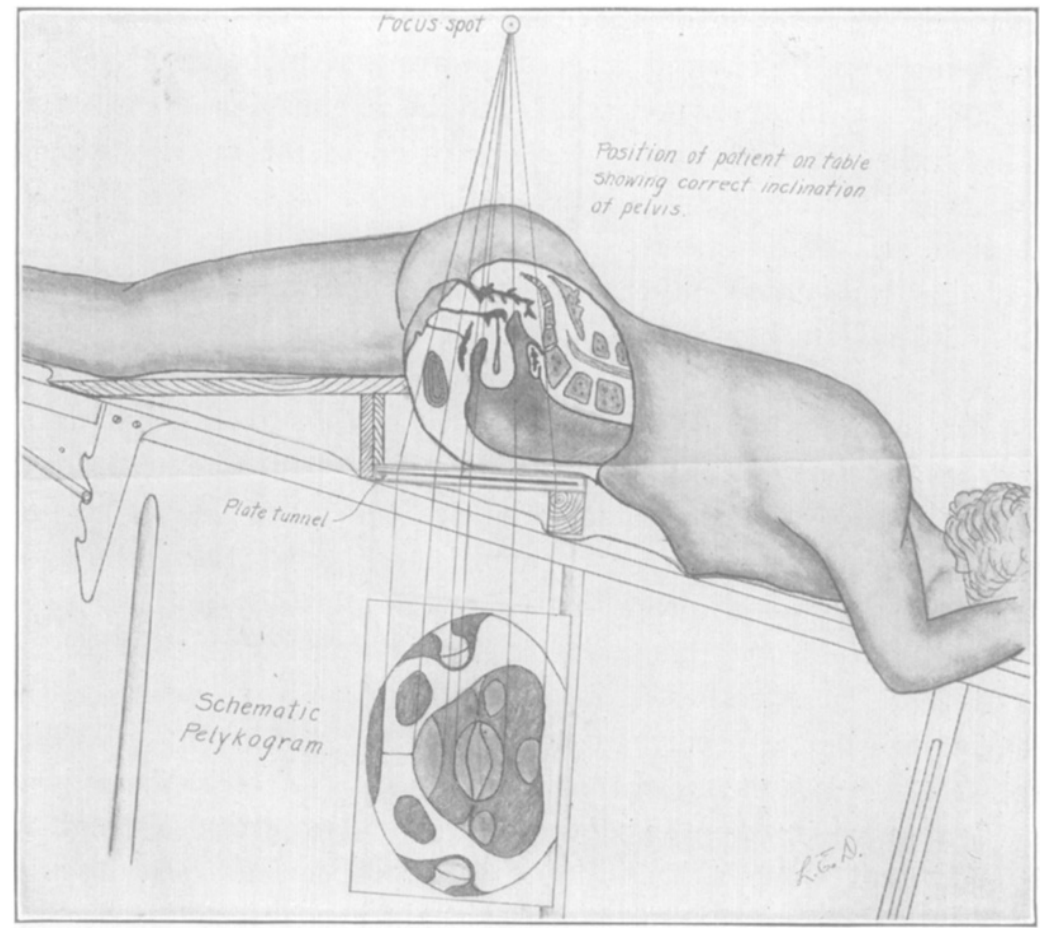

F1o. y.-Correct inclination of pelvis with schematic pelykogram showing theoretical projection of pelvic organs. Partial knee-chest position secured by prone position of patient on frame and a $20^{\circ}$ inclination of the table. 
actual findings, uninfluenced by the $x$-ray study of the plates, are recorded. If the abdomen be opened, the position and condition of the pelvic organs are dictated at the time and the final records of the case together with any removed organs are studied with the plates.

It certainly has been surprising to see how often it has been impossible to determine accurately by the examining finger the exact condition of the pelvic organs. I had failed to realize how much I was depending upon the opening of the abdomen to clear up fine points in diagnosis. From a study of the history together with a thorough pelvic examination it was decided whether the case was an operative one or not. The patients were always examined under anesthesia prior to operation but not so much to determine more accurately the exact pelvic pathology as to confirm the opinion that the case was one for operation. For always the easier method of making the exact diagnosis was ahead of one since everything was to be cleared up after the incision had been made.

All this has been changed since the x-ray of the pelvis has been utilized as an aid to diagnosis. The clinician is placed upon his mettle, so to speak. He carefully weighs his opinion regarding the size and position of the uterus, the tubes and ovaries, if he is to go over his recorded findings and compare them with those of his colleague whose only knowledge of the case is what he sees depicted on the roentgenograms. Renewed interest is aroused in being able to diagnosticate prior to operation what had been considered rather minor pelvic conditions, such as the exact position of the ovaries and tubes, or whether they be free or slightly adherent. At any rate it is a fact that preoperative diagnosis, since the advent of the pelvic $x$-ray, has received a tremendous impetus in the clinic and has greatly improved.

In definitely stating what can be seen in technically good roentgenograms of the pelvis it must be borne in mind that this new field is constantly changing. What could not be seen at the beginning of our work is perfectly apparent today and the same will probably apply to interpretations of plates months from now. However, enough can be made out at the present time to be of great aid in diagnosis.

The pelvic organs are represented on the plate by optical cross seetions at the point of tangency of the projecting rays. (Figs. 8 to 20.) The uterus is nearly always clearly ontlined and one can judge of its position, size and contour. If not drawn to one side by adhesions or displaced hy a neoplasm, so that the ray as it is shot in the axis of the pelvis eatches it fairly, the uterus will show two cross sections, one of the body and one of the isthmus or supracervical portion. It has been found that the isthmus is greatly enlarged and extends more into the broad ligaments in cases of pregnancy than in the nompregnant. So striking is the cliange in the isthmus that by this sign alone it has 
been possible to diagnosticate pregnancy by the x-ray as early as the sixth week and before softening can be determined definitely by the examining finger. The sign is constant from two and one-half months onward, but we have not had sufficient material to date to be too dog-

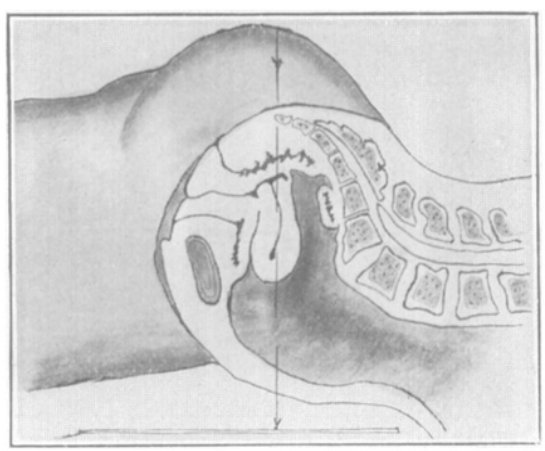

Fig 10.

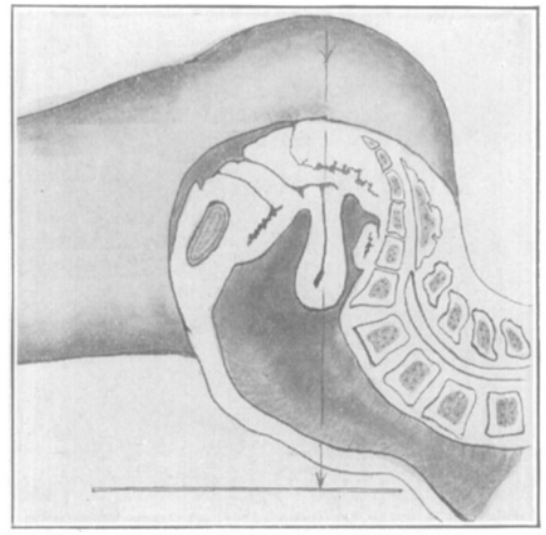

Fig. 11.

Fig. 10.-Insufficient inclination--no gas in vesicouterine pouch. Failure to differentiate anterior surtace of uterus-may or may not project isthmus.

Fig. 11.- I xcessive inclination. Restriction of posterior culdesac with failure to differentiate posterior surface of uterus and appendages.

matic about the presence of this enlargement of the isthmus in every case of early pregnancy.

Irregularities of the uterus produced by fibroids and especially calcification changes in these growths are readily recognized on the

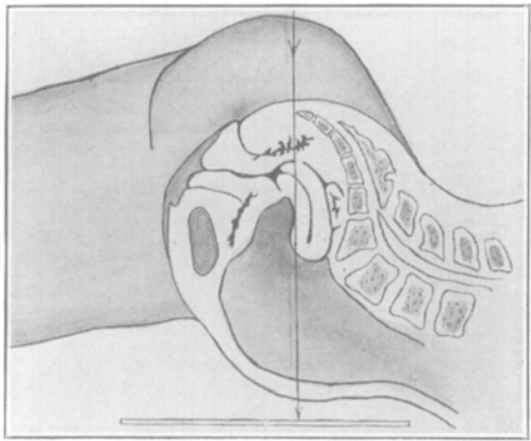

Fig. 12 .

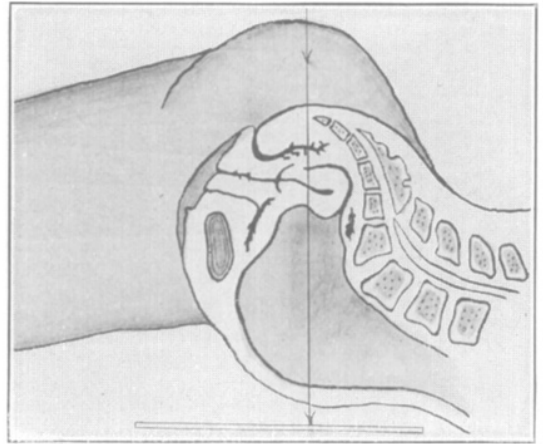

Fig. 13. Jig. 12.-Retrocession. Obliteration of posterior culdesac and excessive width of the anterior
culdesac. Simulates excessive inclination of pelvis.

lig. 13,-Adlierent retroversion. Complete failure to differentiate uterus. Often complicated by adherent bowel sladows.

$x$-ray plate. Through the changes in the isthmus described above it has been found possible to diagnosticate positively the occurrence of pregnancy in a fibroid uterus at a stage when it could not be considered probable from the history and clinical findings. In a num- 
ber of cases where the patient has been exposed to pregnancy and where menstruation is irregular it has been possible to state definitely that the patient was not pregnant because of the size of the uterus and the absence of change in the isthmus.

Since experience has determined jush the right inclination of the

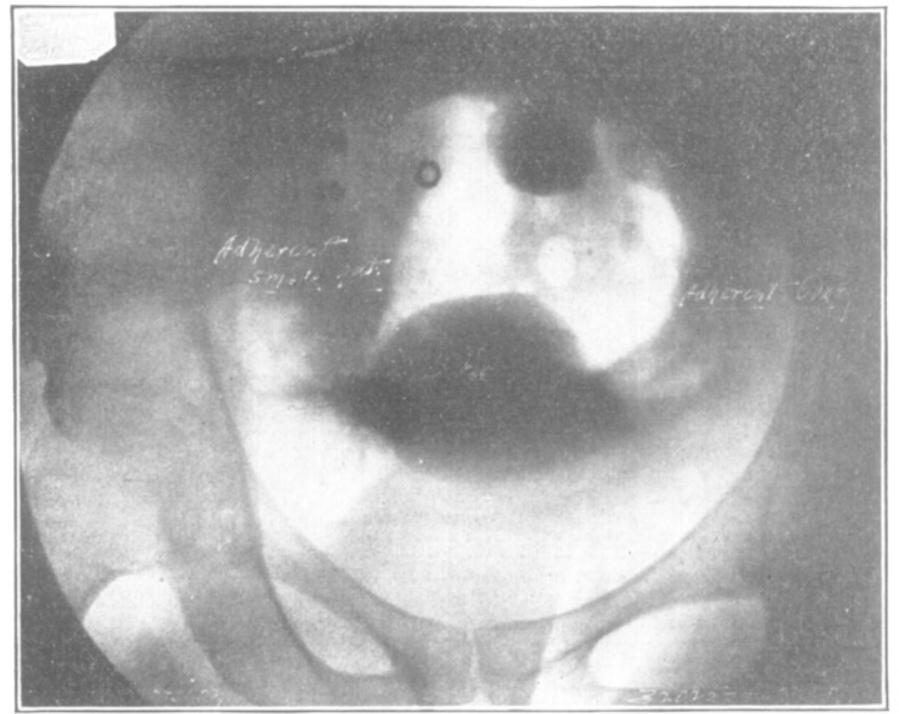

Fig. 14.-32025-Uterus singhly enlarged in the axis of the pelvis, appendages lost in the thickening on the inferior lateral wall of the pelvis and obviously adherent. An example of the spontaneous replacement of a non-adherent retroverted uterus.

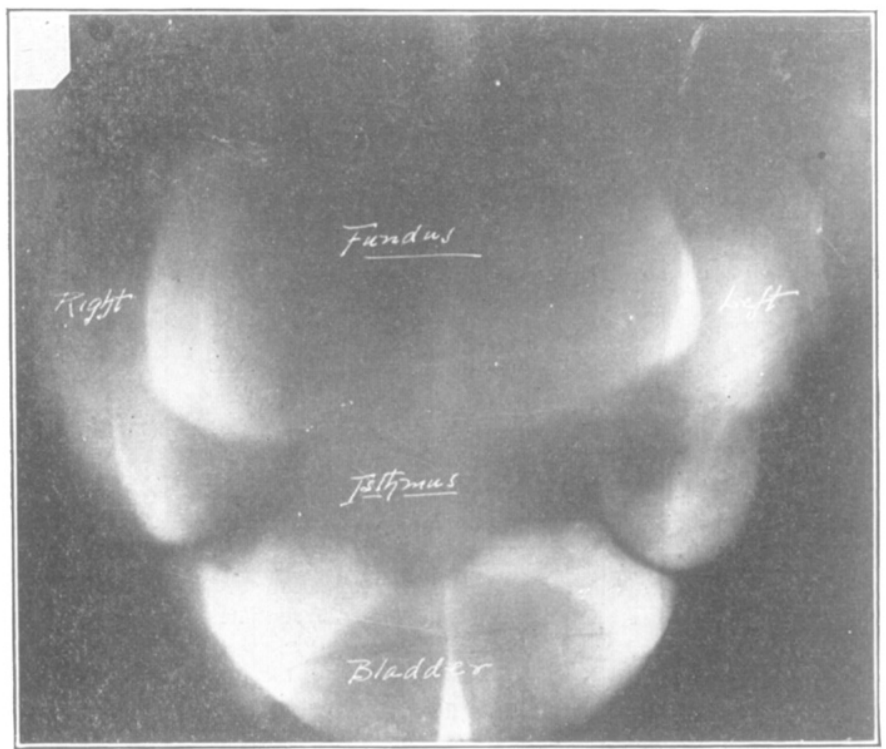

Fig. 15.--30426--Three montbs' pregnancy. The entire upper portion of the pelvis is filler with a rounded shadow representing the fundus of the uterus. Broad expansion of the isthmus, conspicuous round ligaments placed on the stretch, appendages hidden under the shadow of the fundus. 
patient in the prone position on the table to bring about the proper forward and downward displacement of the appendages, normal ovaries and tubes can be shown on the $\mathrm{x}$-ray plates with great regularity. The size of the ovary can be quite accurately estimated and usually it is perfectly possible to differentiate between the free and the adherent organ.

It is not so casy to see normal tubes unless the gas has been passed by the transuterine route when from their distention they are quite clearly made out. Enlarged nodular noncystic tubes are well depicted upon the plates and not infrequently are much better seen than

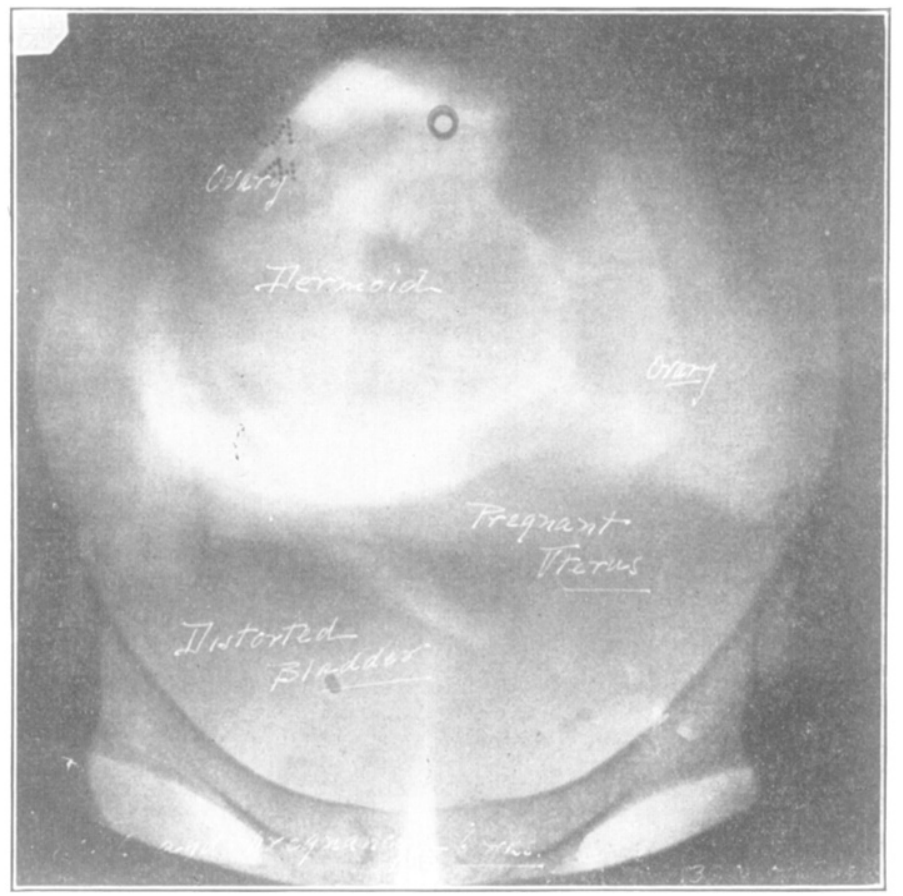

Fig. 16.-32024-Dermoid cysts complicated with six weeks' pregnancy. The uterus is s)ightly entarged with conspicisnus and enlarged isthmus displaced to the left by the large rounded shadow of the dermoid occupying the right posterior portion of the pelvis. The right ovary is displaced upwards and to the right. The left ovary is somewhat enjarged, the bladder partly distended and deformed by pressure.

palpated. Especially is this true when the tubes are adherent to the sides of the pelvis and covered with plastic exudate. In these cases the elinician has long realized the difficulties of palpation and has depended upon the history, thickening in the region of the broad ligaments and deereased uterine movability as diagnostic signs of the condition.

Pus tubes with adhesions to the broad ligaments and bowel coils are particularly well seen on the $x$-ray plates. To be sure the clinician usually has little difficulty in palpating fairly distinct sacular disten- 
tion of the tubes but when the pelvis is the seat of an extensive inflammatory process it is often quite impossible to differentiate the distended tubes. From experience the examiner judges what will be found when the abdomen or posterior culdesae is opened but his differentiation of the contents of the pelvis is not nearly as definite as can be brought out by the x-ray.

Pelvic neoplasms if not so tightly wedged in the pelvis as to prevent the gas from surrounding them are elearly shown by the roentgenograms. Since it is perfectly possible to show the outlines of the liver, spleen and kidneys by pneumoperitoneal x-ray plates of these

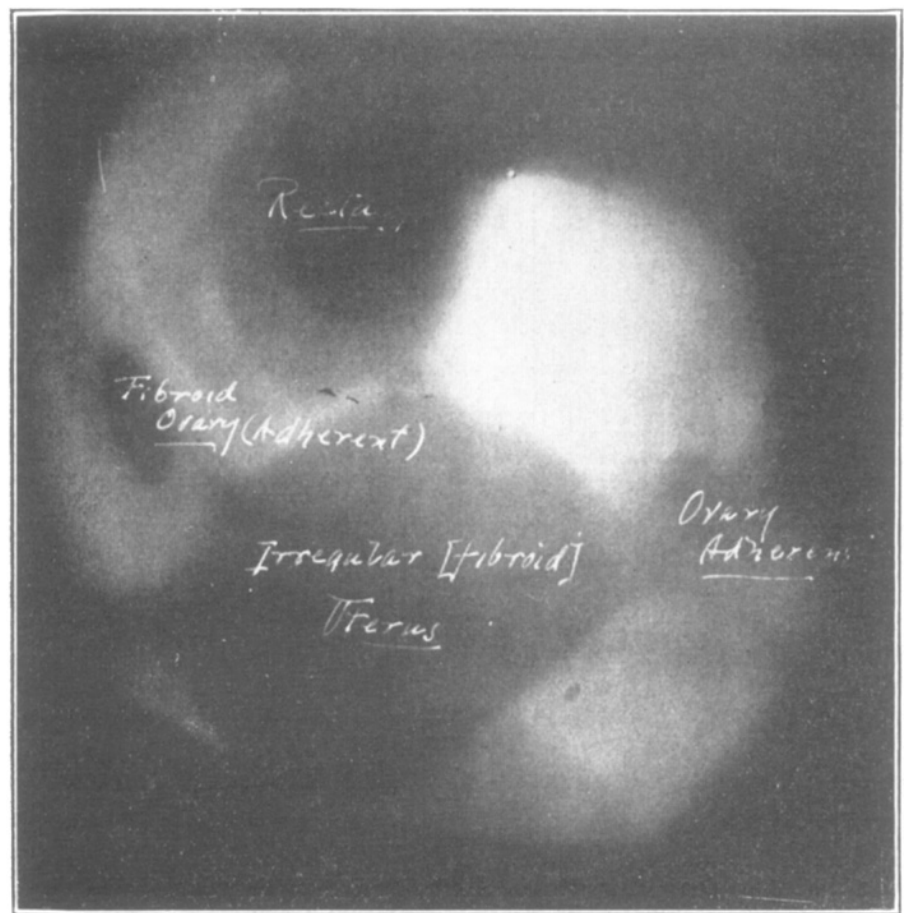

Fig. 17. -3015y_ribtoma of the uterus and bilateral adherent ovaries. The irregularly wilarged uterus occupies the axis of the pelvis, both ovarian shadows are small, lying oin the floor of the pelvis and at a lower level than the fundus. On the left there is some evidence of irregular adhesions. The position of the ovaries on the foor of the pelvis in the modifed knee-chest position is indicative of their fixation by adhesions.

organs, usually it cau be determined by a study of roentgenograms of the pelvis and upper abdomen whether neoplasms arise from the pelvis or elsewhere and this means of diagnosis entails far less pain or discomfort than catheterization of the ureters or other diagnostic measures.

The above will suffice to show the possibilities of the pneumoperitoneal $\mathrm{x}$-ray, since detailed clinical and roentgenographic findings will be dealt with in a subsequent paper. While pointing out what has been and can be accomplished by the new diagnostic procedure, it must not be thought that the idea is to belittle or do away with biman- 
ual pelvic examination. In quite a proportion of cases information derived from this source proved far more accurate than that furnished by the x-ray. This was to be expected for bimanual examination has been practiced for years, while this kind of $x$-ray work is new and confined to only a few hundred observations. In fact nothing is to be

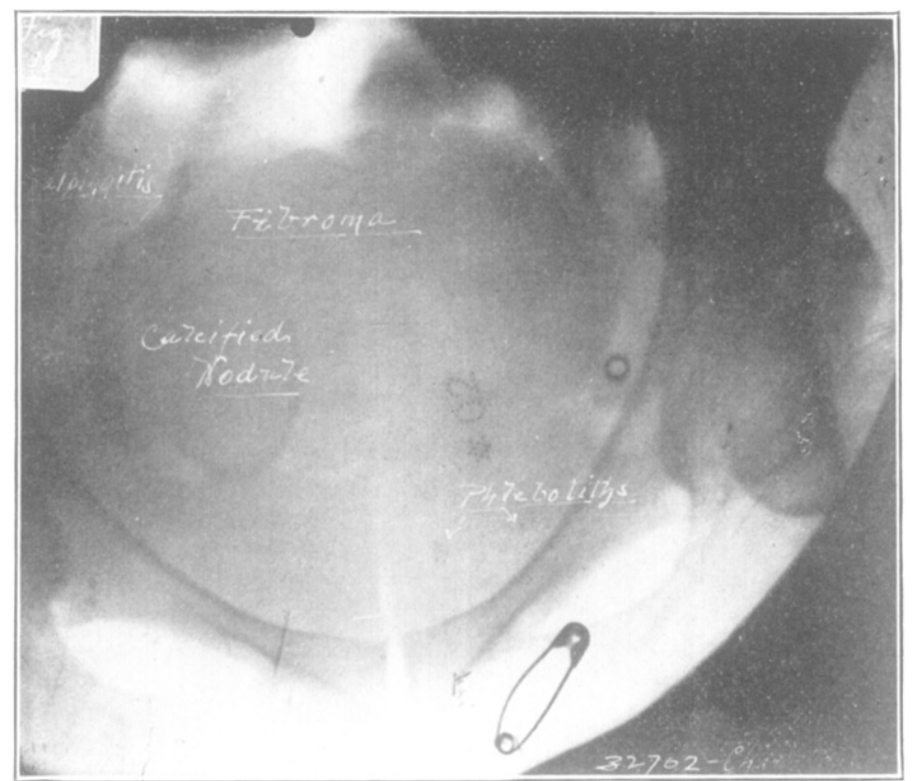

Fig. 18.-32702-Large fibroma occupying the upper portion of the pelvis and showing a nodular shadow representing calcification of a fibromatous nodule. An enlarged tube is seen jresenting from behind the mass in the right posterior portion of the pelvis.

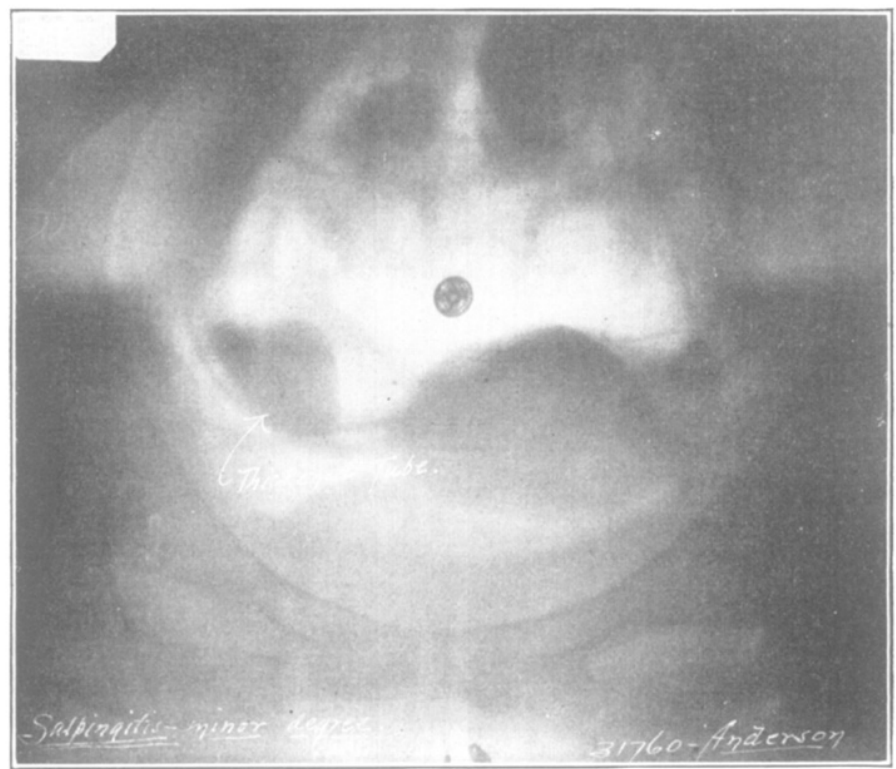

Fir. 19.-31760--Salpingitis, minor degree. Insufficient inclination of the patient, producing a narrow anterior culdesac. The right tube can be distinctly recugnized as a thickened cord. Otherwise negative. 
gained, on the contrary much will be lost, by raising the issue of antagonism between the two diagnostic methods. Just as it is scarcely fair to demand that the roentgenologist interpret the pelvic $x$-ray plate without any knowledge of the case, so it is asking rather too much of the elinician in certain cases to state definitely the condition of the pelvic organs from bimanual examination alone. The rule of making separate diagnoses has been followed by us deliberately in order to gain the most knowledge from our different diagnostic meth-

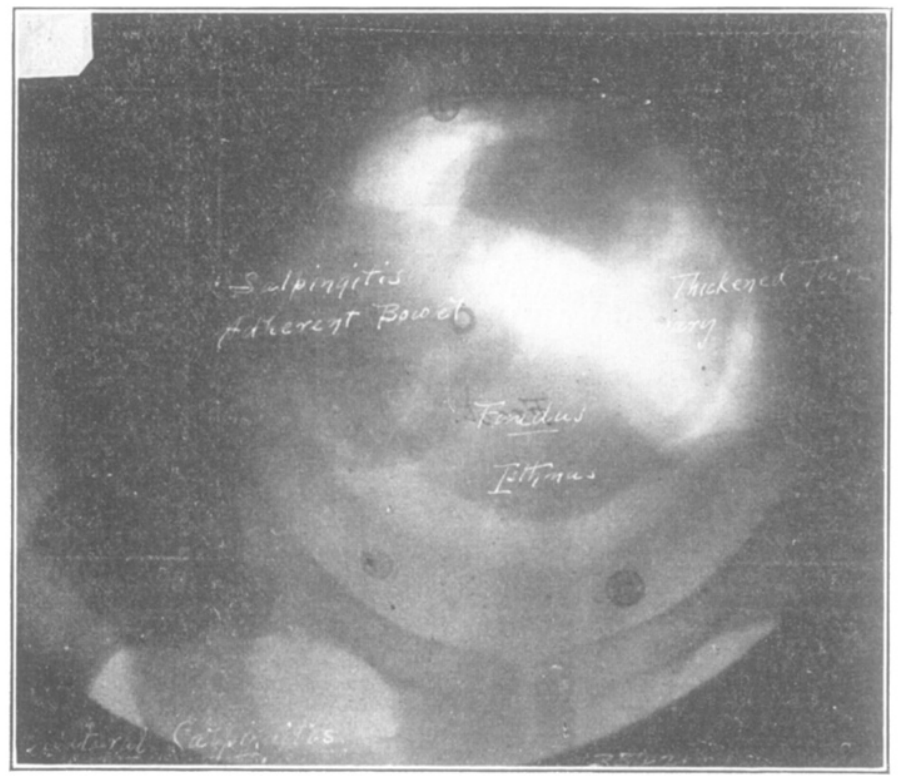

Fig. 20.-33221-Bilateral salpingitis, The fundus of the uterus is apmoximately in the normal position, flanked on either side $b y$ appendages of increased size and density, more marked on the right. The included gas shadows in the right side prove the presence of adherent bowel coils over the appendage.

ods. From now on it is planned to give due consideration to the other's findings in order that the most accurate diagnosis can be arrived at by the fullest cooperation.

It must be borne in mind that only about 300 patients have been sufjected to the pneumoperitoneal $x$-ray. This means that the work, compared with tens of thousands of gastrointestinal x-rays, is just beginning. Greater study and experience will lead to such accuracy of diagnosis that it will be considered as faulty in doubtful cases to do pelvic work without a pneumoperitoneal x-ray, as to operate upon the stomach or upper abdomen without a roentgenogram.

Since it cannot be expected of the roentgenologist that he make clinieal examinations, probably perfected diagnostic technic will come from the clinieian who has made himself master of the pneumoperitoneal pelvic roentgenogram. With his knowledge of pelvic pathology which comes from his everyday work, he is better equipped than any 
one to interpret the pelvic plate provided he is willing to give up the necessary time and study to its interpretation. At least this is not too much to hope in the case of the young obstetrician and gynecologist just starting on his special work. For I am convinced that if we are to demonstrate that there is real need for our specialty, it must be through developing preoperative diagnosis. It should be the aim of the gynecologist by accurate diagnosis to state with ever greater and greater positiveness when an operation is indicated and when the symptoms can be made to disappear by other methods of treatment.

\section{SUMMIARY AND CONCLUSTONS}

1. The pneumoperitoneal $\mathrm{x}$-ray is a great aid to accurate obstetric and gynecologic diagnosis.

2. In suitable cases and with the proper technic, gas inflation is free from danger.

3. The apparatus for gas inflation and pelvic roentgenography is simple and inexpensive and can be used in any obstetric or gynecologic examining room.

4. The method should not be used in cases of acute pelvic inflammation or when disturbances of circulation may arise from sudden abdominal distention.

5. Since carbon dioxide gas is absorbed within lnalf an hour, it is preferable to nxygen gas for inflation, since the latter gas may not be absorbed for days.

6. Whenever possible the transuterine should be chosen in preference to the transperitoneal route for the introduction of the gas because of the valuable information it furnishes regarding the permeability of the fallopian tubes.

7. Excessive quantities of gas cause great pain. Experience has shown that for the ordinary case 1000 cubic centimeters of gas will cause only moderate discomfort and are sufficient for good roentgenograms.

8. With the proper position (partial knee-chest with tilted table) and the ray directed perpendicularly to the plate in the axis of the pelvis the pelvic organs are clearly shown by roentgenography.

9. Experience with pneumoperitoneal pelvic roentgenography will enable the observer to diagnosticate with great accuracy the pathologic changes in the pelvic organs.

10. There is every indication that pregnancy can be diagnosticated as early as the sixth week by the pneumoperitoneal x-ray.

11. Bimanual pelvic examination and pelvic pneumoperitoneal roentgenography are not antagonistic diagnostic methods. Each is valuable and their value is enhanced if they be used in conjunction, each acting as a check upon the other. 